Revista de Matemática: Teoría y Aplicaciones 2015 22(1) : 31-47

CIMPA - UCR ISSN: 1409-2433 (PRINT), 2215-3373 (ONLINE)

\title{
LOCAL CONVERGENCE OF EXACT AND INEXACT NEWTON'S METHODS FOR SUBANALYTIC VARIATIONAL INCLUSIONS
}

\author{
CONVERGENCIA LOCAL DE LOS MÉTODOS DE \\ NEWTON EXACTO E INEXACTO PARA \\ INCLUSIONES VARIACIONALES SUBANALÍTICAS
}

\author{
Catherine Cabuzel* Alain Pietrus ${ }^{\dagger}$ \\ STEEVE Burnet B $^{\ddagger}$
}

Received: 20/Jun/2013; Revised: 22 Oct 2014;

Accepted: 29 Oct 2014

\footnotetext{
*Laboratoire LAMIA-EA 4540, Université des Antilles et de la Guyane, Département de Mathématiques et Informatique, Campus de Fouillole, F-97159 Pointe-à-Pitre, France. E-mail: catherine.zebre@univ-ag.fr

${ }^{\dagger}$ Misma dirección que/same address as C. Cabuzel. E-mail: apietrus@univ-ag.fr

${ }^{\ddagger}$ Misma dirección que/same address as C. Cabuzel. E-mail: sburnet@martinique.univ-ag.fr
} 


\begin{abstract}
This paper deals with the study of an iterative method for solving a variational inclusion of the form $0 \in f(x)+F(x)$ where $f$ is a locally Lipschitz subanalytic function and $F$ is a set-valued map from $\mathbb{R}^{n}$ to the closed subsets of $\mathbb{R}^{n}$. To this inclusion, we firstly associate a Newton then secondly an Inexact Newton type sequence and with some semistability and hemistability properties of the solution $x^{*}$ of the previous inclusion, we prove the existence of a sequence which is locally superlinearly convergent.
\end{abstract}

Keywords: set-valued mapping; variational inclusion; semistability; hemistability; subanalytic function; Newton's method; inexact Newton's method.

\title{
Resumen
}

En este artículo se estudia un método iterativo para resolver una inclusión variacional de la forma $0 \in f(x)+F(x)$, donde $f$ es una función punto-conjunto, subanalítica, localmente Lipschitz y $F$ es una función multivaluada de $\mathbb{R}^{n}$ en los subconjuntos cerrados de $\mathbb{R}^{n}$. A esta inclusión se le asocia, en primer lugar, una sucesión tipo Newton y, posteriormente una sucesión tipo Newton inexacto. Bajo algunas propiedades de semi-estabilidad y hemi-estabilidad de la solución $x^{*}$ de la inclusión variacional, se demuestra la existencia de una sucesión que es superlinealmente localmente convergente.

Palabras clave: función multivaluada; inclusión variacional; semi-estabilidad; hemi-estabilidad; función subanalítica; método de Newton; método de Newton inexacto.

Mathematics Subject Classification: 49J53, 47H04, 65K10, $14 \mathrm{P} 15$.

\section{Introduction}

The variational inclusions were introduced by Robinson $[34,36]$ as an abstract model of many problems encountered in various fields such as mathematical programming, engineering [20], optimal control. Robinson showed in particular that complementarity problems can be expressed in terms of variational inclusions by the use of the normal cone to a set. Consequently, the theory that concerns the solving of these inclusions has been developed and the number of publications in this area has increased for these two last decades. 
In this paper, our goal is to solve the variational inclusion

$$
0 \in f(x)+F(x)
$$

where $f: X \subset \mathbb{R}^{n} \rightarrow \mathbb{R}^{n}$ is locally Lipschitz and subanalytic on a neighborhood $\Omega \subset X$ of $x^{*}$, solution of (1) and $F: X \subset \mathbb{R}^{n} \rightrightarrows \mathbb{R}^{n}$ is a set-valued map with closed graph.

Several iterative methods have been presented to solve (1); one which is more original was introduced by Dontchev $[15,17]$ in which he considered a Newton type sequence where $f$ is a function whose first order Fréchetderivative $f^{\prime}$ satisfies a Lipschitz condition; in [16], he showed the stability of the method. Following these works, Pietrus [31, 32] obtained similar results when $f^{\prime}$ verifies an Hölder condition obtained similar results.

Starting with Dontchev's work, various iterative methods have been introduced in the last decade. They consist in generating an iterative sequence $\left(x_{k}\right)$ obtained by subsequently solving implicit subproblems of the form $0 \in A\left(x_{k}, x_{k+1}\right)+F\left(x_{k+1}\right)$, where $A$ denotes some approximation of the mapping f. For the convenience of the reader, we mention some of these works. For example, using a second-degree Taylor polynomial expansion of $f$ at $x_{k}$, Geoffroy, Hilout and Pietrus in [22] introduced a method involving the second order Fréchet-derivative and, they obtained a cubic convergence when $f^{\prime \prime}$ is Lipschitz. In [24], they proved the stability of the method and in [23] it is showed that the previous method is superquadratic when $f^{\prime \prime}$ satisfies a Hölder condition. Jean-Alexis presented in [27] a method without second order Fréchet-derivative, which is also cubically convergent. Lately, the authors in [11] proposed a generalization of these methods by taking more iterates.

In the case where the single-valued part of the variational inclusion is nonsmooth, the previous methods are not valid. That led us to introduce and investigate in [10] a Newton type algorithm to solve (1) when $f$ is a subanalytic and locally Lipschitz function. This Newton type algorithm is the so-called subanalytic Newton method (SNM) which can be described in the following way: for the current iterate $x_{k} \in \mathbb{R}^{n}$, the next iterate $x_{k+1}$ is computed as a solution of the pseudo-linearized Variational Inclusion at $x_{k}$ :

$$
0 \in f\left(x_{k}\right)+\Delta f\left(x_{k}\right)\left(x_{k+1}-x_{k}\right)+F\left(x_{k+1}\right)
$$

where $\Delta f\left(x_{k}\right) \in \partial^{\circ} f\left(x_{k}\right)$ and $\partial^{\circ} f\left(x_{k}\right)$ denotes the Clarke Jacobian of $f$ at the point $x_{k}$. In this context we obtained with some metric regularity as- 
sumptions the existence of superlinear convergent sequences defined by (2).

Let us point out that all these methods have been studied provided a pseudo-Lipschitz property is satisfied for the set-valued map $(f+F)^{-1}$ or one of its approximation and a fixed point theorem is used. For more details on this property and the fixed point theorem evocated, the reader can refer, for example to $[1,2,14,18,19,29,30,38]$.

It is possible to obtain interesting results without the use of the previous concepts. Recently in $[7,8,9]$, the authors studied inclusion (1) in the smooth case by using an assumption which is directly connected to a solution: the semistability concept. This concept has been introduced by Bonnans [6] for variational inequalities. A solution $x^{*}$ of a variational inclusion is said to be semistable if, given a small perturbation on the left-hand side of (1), a solution $x$ of the perturbed variational inclusion that is sufficiently close to $x^{*}$ is such that the distance of $x$ to $x^{*}$ is of the order of the magnitude of the perturbation.

Recently, Izmailov and Solodov in [26], used the concept of semistability and another concept which is the hemistability in order to study the existence and the convergence of the following inexact Josephy-Newton method,

$$
0 \in f\left(x_{k}\right)+f^{\prime}\left(x_{k}\right)\left(x_{k+1}-x_{k}\right)+\omega_{k}+F\left(x_{k+1}\right),
$$

where $\omega_{k}$ is a perturbation term and they obtained with some assumptions superlinear and quadratic convergence for the sequence $\left(x_{k}\right)$.

Our goal in this paper is to show that we can find similar conclusions in the nonsmooth case when the function $f$ is Lipschitz and subanalytic.

The paper is organized as follows: in Section 2, we collect some definitions regarding semianalytic, subanalytic functions, differentiability results, semistability and hemistability of solutions of variational inclusions. In Section 3, we study the subanalytic Newton method and in Section 4, we study the inexact subanalytic Newton method. In both cases we obtain at least a superlinear convergence.

\section{Preliminaries}

Let us begin with some notations we use in the whole document. We denote by $x^{*}$ a solution of (1), by $\|$.$\| the norm in \mathbb{R}^{n}$; for a point $x \in X$ and a set $C \subset X$, the distance of $x$ to the set $C$ is defined by $\operatorname{dist}(x, C)=$ $\inf \{\|x-y\|, y \in C\}$, the closed ball centered at $x$ with radius $r$ by $B_{r}(x)$. Let $\Lambda: X \rightrightarrows Y$ be a set-valued map, we denote by $\operatorname{graph} \Lambda=\{(x, y) \in$ 
$X \times Y, y \in \Lambda(x)\}$ the graph of $\Lambda$, by $\Lambda^{-1}(y)=\{x \in X, y \in \Lambda(x)\}$ the inverse of $\Lambda$ and $\mathcal{L}(X, Y)$ is the space of bounded linear operators from $X$ into $Y$.

\subsection{Semianalytic and subanalytic functions}

The first work on subanalytic sets seems to be attributed to Gabrielov [21] in which we can find the definition 2.2, but Hironaka [25] was the first to produce a complete and simple theory on subanalytic sets; then other authors like Dedieu [13] continued to simplify the ideas on this topic. We extracted the following definitions, properties and examples from Dedieu's paper. However, the reader who wants to have a more extensive view on the algebraic geometry can refer to this nonexhaustive list of references as Bierstone and Milman [3], Bochnak, Coste and Roy [4] or Lojasiewicz [28].

Definition 2.1 A subset $X$ of $\mathbb{R}^{n}$ is semianalytic if for each a $\in \mathbb{R}^{n}$ there is a neighborhood $U$ of $a$ and real analytic functions $f_{i, j}$ on $U$ such that

$$
X \cap U=\bigcup_{i=1}^{r} \bigcap_{j=1}^{s_{i}}\left\{x \in U \mid f_{i, j}(x) \varepsilon_{i, j} 0\right\}
$$

where $\varepsilon_{i, j} \in\{<,>,=\}$.

When $U=\mathbb{R}^{n}$ and the $f_{i, j}$ are polynomials, one says that $X$ is semialgebraic.

Definition 2.2 A subset $X$ of $\mathbb{R}^{n}$ is subanalytic if each point a $\in \mathbb{R}^{n}$ admits a neighborhood $U$ such that $X \cap U$ is a projection of a relatively compact semianalytic set: there is a semianalytic bounded set $A$ in $\mathbb{R}^{n+p}$ such that $X \cap U=\Pi(A)$ where $\Pi: \mathbb{R}^{n+p} \rightarrow \mathbb{R}^{n}$ is the projection.

Definition 2.3 Let $X$ be a subset of $\mathbb{R}^{n}$. A function $f: X \rightarrow \mathbb{R}^{m}$ is semianalytic (resp. subanalytic) if its graph is semianalytic (resp. subanalytic).

Let us note that the class of semianalytic sets is stable for elementary set operations (finite union, finite intersection, set difference), the closure, the interior and the connected components of a semianalytic set are semianalytic. The same properties hold for subanalytic sets. The image of a bounded semianalytic set by a semianalytic function is not necessarily 
semianalytic. Consequently, the class of semianalytic functions is not stable under algebraic operations (sum, product, composition see [28], page 133). Subanalytic functions have been introduced for this reason: if $X$ is a subanalytic and relatively compact set the image of $X$ by a subanalytic function is subanalytic (see [3], page 16).

In the references given above, we can find interesting examples of subanalytic functions in relation with optimization like the distance function or the supremum of a finite family of subanalytic continuous functions.

\subsection{Differentiability results}

Proposition 2.1 (canonical approximation) If $f: X \subset \mathbb{R}^{n} \rightarrow \mathbb{R}^{n}$ is a subanalytic locally Lipschitz mapping then for all $x \in X$

$$
\left\|f(x+d)-f(x)-f^{\prime}(x ; d)\right\|=o_{x}(\|d\|) .
$$

where $f^{\prime}(x ; d)$ is the directional derivative of $f$ at $x$ in the direction $d$.

We know by [39] that, the subanalytic function $t \rightarrow o_{x}(t)$ admits a Puiseux development; so there are a positive constant $c$, a real number $\varepsilon>$ 0 and a rational number $\gamma>0$ that come from the Puiseux development such that $\left\|f(x+d)-f(x)-f^{\prime}(x ; d)\right\|=c\|d\|^{\gamma}$ whenever $\|d\| \leq \varepsilon$.

Definition 2.4 Let $f: \mathbb{R}^{n} \rightarrow \mathbb{R}^{m}$ be a locally Lipschitz continuous function.

The limiting Jacobian of $f$ at $x \in \mathbb{R}^{n}$ is defined as

$$
\partial f(x)=\left\{A \in \mathcal{L}\left(\mathbb{R}^{n}, \mathbb{R}^{m}\right): \exists u^{k} \in D ; f^{\prime}\left(u^{k}\right) \rightarrow A, k \rightarrow+\infty\right\}
$$

where $D$ denotes the points of differentiability of $f$ and the Clarke Jacobian $\partial^{\circ} f(x)$ of $f$ at $x \in \mathbb{R}^{n}$ is a subset of $X^{*}$, defined as the closed convex hull of $\partial f(x)$ (see [12], page 70$)$.

A consequence of the Lipschitz property is that the Clarke Jacobian of $f$ is a nonempty compact convex set. Moreover we have the following proposition:

Proposition 2.2 [5] Let $f: \mathbb{R}^{n} \rightarrow \mathbb{R}^{n}$ be locally Lipschitz and subanalytic, there exists a positive rational number $\gamma$ such that:

$$
\|f(y)-f(x)-\Delta f(y)(y-x)\| \leq C_{x}\|y-x\|^{1+\gamma}
$$

where $\Delta f(y)$ is any element of $\partial^{\circ} f(y)$ and $C_{x}$ is a positive constant. 


\subsection{Semistable and hemistable solutions}

Definition 2.5 A solution $x^{*}$ of (1) is said to be semistable if for any $r \in \mathbb{R}^{n}$ close enough to zero, any solution $x(r)$ of the perturbed variational inclusion

$$
r \in f(x)+F(x)
$$

close enough to $x^{*}$ satisfies the estimate

$$
\left\|x(r)-x^{*}\right\|=O(\|r\|)
$$

It is well-known, that semistability only gives information on the rate of convergence of trajectories which converge to $x^{*}$ but does not guarantee solvability of the problem (1) for a trajectory $\left(x_{k}\right)$ close to $x^{*}$. In order to obtain existence, we need the following notion.

Definition 2.6 Let $f: \mathbb{R}^{n} \rightarrow \mathbb{R}^{n}$ be a smooth function. A solution $x^{*}$ of (1) is said to be hemistable if for any $x \in \mathbb{R}^{n}$ close enough to $x^{*}$, the variational inclusion

$$
0 \in f(x)+f^{\prime}(x) \eta+F(x+\eta)
$$

has a solution $\eta(x)$ such that $\eta(x) \rightarrow 0$ as $x \rightarrow x^{*}$.

Generally, none of the two properties of semistability and hemistability is implied by the other. In [6], it has been briefly shown that a sufficient condition for both to be satisfied is the strong regularity condition of Robinson [35].

The above condition has no sense for nonsmooth functions, it is the reason why we extend this notion to Lispchitz function which admits a subgradient. In this case, we replace the above definition by the following which is more general.

Definition 2.7 Let $f: \mathbb{R}^{n} \rightarrow \mathbb{R}^{n}$ be a Lipschitz continuous function (not necessarily smooth). A solution $x^{*}$ of (1) is said to be hemistable if for any $x \in \mathbb{R}^{n}$ close enough to $x^{*}$ and $\Delta f(x) \in \partial^{\circ} f(x)$, the variational inclusion

$$
0 \in f(x)+\Delta f(x) \eta+F(x+\eta)
$$

has a solution $\eta(x)$ such that $\eta(x) \rightarrow 0$ as $x \rightarrow x^{*}$.

\section{Remark 2.1}

- This definition is valid in the case where $f$ is a semi-smooth function (see [33]) in particular when $f$ is Lipschitz subanalytic.

- In the case of smooth functions both definitions coincide. 


\section{Convergence analysis of the subanalytic Newton type method}

In [10], we obtained the existence of Newton type sequences for variational inclusions and we prove the superlinear convergence of these trajectories. In this work the result has been obtained with a more theoretical assumption of metric regularity on the set valued map. For more details on this topic and applications, the reader could be referred to $[1,2,18,14,29,30,37,38]$ and the references therein.

In the rest of the paper we suppose that $f$ is a Lipschitz and subanalytic function.

Our aim here is to give some results on the rate of convergence of these sequences using semistability or hemistability of the solution instead of the metric regularity. We start with a first result which gives the rate of convergence when we suppose the existence and the convergence of the sequence.

Theorem 3.1 Let $x^{*}$ be a semistable solution of (1), and let $\left(x_{k}\right)_{k \geq 0}$ defined by (2) converges towards $x^{*}$ then the convergence is superlinear.

\section{Proof:}

We can write the Newton step (2) by

$$
r_{k} \in f\left(x_{k+1}\right)+F\left(x_{k+1}\right)
$$

where $r_{k}=f\left(x_{k+1}\right)-f\left(x_{k}\right)-\Delta f\left(x_{k}\right)\left(x_{k+1}-x_{k}\right)$ and $\Delta f\left(x_{k}\right) \in$ $\partial^{\circ} f\left(x_{k}\right)$.

As in $[5,10]$, denoting $C_{*}$ the biggest value of the constant $C_{x}$ in an appropriate neighborhood $\Omega$ of $x^{*}$, the inequality (3) in Proposition 2.2 remains valid replacing $C_{x}$ by $C_{*}$. Thus, from the Proposition 2.2 we obtain the existence of a rational constant $\gamma>0$ such that $\left\|r_{k}\right\|=$ $O\left(\left\|x_{k+1}-x_{k}\right\|^{1+\gamma}\right)=o\left(\left\|x_{k+1}-x_{k}\right\|\right)$.

The semistability of $x^{*}$ implies that

$$
\left\|x_{k+1}-x^{*}\right\|=O\left(\left\|r_{k}\right\|\right)=o\left(\left\|x_{k+1}-x_{k}\right\|\right)=o\left(\left\|x_{k+1}-x^{*}\right\|+\left\|x_{k}-x^{*}\right\|\right),
$$

hence $x_{k+1}-x^{*}=o\left(x_{k}-x^{*}\right)$ and we obtain the superlinear convergence of $\left(x_{k}\right)$.

In the previous theorem we suppose the existence and the convergence of the sequence and obtain the rate of convergence, the following result is 
more general in the sense that we also prove existence of trajectories using in addition the hemistability assumption.

Theorem 3.2 Let $x^{*}$ be a semistable and a hemistable solution of (1). Then there exists $\varepsilon>0$ such that for any starting point $x_{0} \in \mathbb{R}^{n}$ close enough to $x^{*}$, there exists a trajectory $\left(x_{k}\right)_{k \geq 0}$ defined by (2) and $\| x_{k+1}-$ $x_{k} \| \leq \varepsilon$. Moreover this trajectory converges towards $x^{*}$ and the rate of convergence is superlinear.

\section{Proof:}

The semistability gives the existence of $c_{1}>0$ and $c_{2}>0$ such that for any $r \in \mathbb{R}^{n}$ and any solution $x(r)$ solution of (4), satisfying $\left\|x(r)-x^{*}\right\| \leq c_{1}$, one has $\left\|x(r)-x^{*}\right\| \leq c_{2}\|r\|$.

Let us remark that if $\left\|x_{k}-x^{*}\right\|$ is sufficiently small, the hemistability property allows to find a solution $x_{k+1}$ of (2) such that $\left\|x_{k+1}-x^{*}\right\|$ is small. For this reason, if we take $\varepsilon_{0} \leq \min \left(c_{1}, \frac{1}{5} c_{2}\right)$, from the hemistability condition it holds that for some $\varepsilon \in\left(0, c_{1}\right)$ sufficiently small, $\left\|x_{k}-x^{*}\right\| \leq \frac{4}{5} \varepsilon$ implies the existence of $x_{k+1}$ solution of (2) such that $\left\|x_{k+1}-x^{*}\right\| \leq \varepsilon_{0}$.

It is easy to see that $x_{k+1}$ is also solution of the equation $r_{k} \in f\left(x_{k+1}\right)+$ $F\left(x_{k+1}\right)$ with $r_{k}:=f\left(x_{k+1}\right)-f\left(x_{k}\right)-\Delta f(x)\left(x_{k+1}-x_{k}\right)$.

From Proposition 2.2, we obtain the existence of a rational constant $\gamma>0$ such that $\left\|r_{k}\right\|=O\left(\left\|x_{k+1}-x_{k}\right\|^{1+\gamma}\right)$. Reducing $\varepsilon_{0}$ and $\varepsilon$ if necessary, we obtain $\left\|x_{k+1}-x_{k}\right\|<1$ and

$$
\left\|r_{k}\right\| \leq \frac{1}{5 c_{2}}\left\|x_{k+1}-x_{k}\right\| .
$$

As $\varepsilon_{0} \leq c_{1}$, the semistability property implies that

$$
\left\|x_{k+1}-x^{*}\right\| \leq c_{2}\left\|r_{k}\right\| \leq \frac{1}{5}\left\|x_{k+1}-x_{k}\right\| \leq \frac{1}{5}\left\|x_{k+1}-x^{*}\right\|+\frac{1}{5}\left\|x_{k}-x^{*}\right\|,
$$

hence

$$
\left\|x_{k+1}-x^{*}\right\| \leq \frac{1}{4}\left\|x_{k}-x^{*}\right\|
$$

which yields

$$
\left\|x_{k+1}-x_{k}\right\| \leq\left\|x_{k+1}-x^{*}\right\|+\left\|x_{k}-x^{*}\right\| \leq \frac{1}{4}\left\|x_{k}-x^{*}\right\|+\left\|x_{k}-x^{*}\right\| \leq \varepsilon .
$$

This completes the proof, the superlinear convergence coming from Theorem 3.1. 


\section{Convergence analysis of the subanalytic inexact Newton type method}

The aim of this section is to study the behavior of the Newton method considering some particular perturbations. The inexact Newton method we consider is of the following form

$$
0 \in f\left(x_{k}\right)+\Delta f\left(x_{k}\right)\left(x_{k+1}-x_{k}\right)+\omega_{k}+F\left(x_{k+1}\right)
$$

where $\Delta f\left(x_{k}\right) \in \partial^{\circ} f\left(x_{k}\right)$ and $\omega_{k} \in \mathbb{R}^{n}$ is a perturbation term.

Theorem 4.1 Let $x^{*}$ be a semistable solution of (1). Let a sequence $\left(x_{k}\right)$ be convergent to $x^{*}$, and assume that $x_{k+1}$ satisfies (5) for each $k=0,1, \ldots$ with some $\omega_{k} \in \mathbb{R}^{n}$ such that

$$
\left\|\omega_{k}\right\|=o\left(\left\|x_{k+1}-x_{k}\right\|+\left\|x_{k}-x^{*}\right\|\right)
$$

Then the rate of convergence is superlinear. Moreover if $\left\|\omega_{k}\right\|=$ $O\left(\left\|x_{k+1}-x_{k}\right\|^{2}+\left\|x_{k}-x^{*}\right\|^{2}\right)$, the order of convergence is equal to $\min \{2,1+$ $\gamma\}$ where $\gamma$ is the rational number given in Proposition 2.2.

\section{Proof:}

We can remark that if for some $k x_{k}=x^{*}$, then from the semistability of $x^{*}$ we obtain that $x_{m}=x^{*}$ for all $m \geq k$ and the proof of the theorem easily follows. For the rest of the proof we suppose that $x_{k} \neq x^{*}$ for all $k$.

We can note that for each $k, x_{k+1}$ is a solution of the inclusion $r_{k} \in$ $f(x)+F(x)$ with

$$
r_{k}=f\left(x_{k+1}\right)-f\left(x_{k}\right)-\Delta f\left(x_{k}\right)\left(x_{k+1}-x_{k}\right)-\omega_{k} .
$$

Using Proposition 2.2 and (6), there exist $C_{*}$ and a rational number $\gamma$ such that

$$
\left\|r_{k}\right\| \leq C_{*}\left\|x_{k+1}-x_{k}\right\|^{1+\gamma}+\left\|\omega_{k}\right\|=o\left(\left\|x_{k+1}-x_{k}\right\|+\left\|x_{k}-x^{*}\right\|\right) .
$$

The semistability of $x^{*}$ implies that

$$
\begin{aligned}
\left\|x_{k+1}-x^{*}\right\| & =O\left(\left\|r_{k}\right\|\right)=o\left(\left\|x_{k+1}-x_{k}\right\|\right)+o\left(\left\|x_{k}-x^{*}\right\|\right) \\
& =o\left(\left\|x_{k+1}-x^{*}\right\|+\left\|x_{k}-x^{*}\right\|\right)
\end{aligned}
$$

and we obtain the superlinear convergence. 
The rest of the proof is very close to the one given in [26]. We give it here for the paper to be self-contained.

If $\gamma \leq 1$ then $\left\|r_{k}\right\|=O\left(\left\|x_{k+1}-x^{*}\right\|^{1+\gamma}+\left\|x_{k}-x^{*}\right\|^{1+\gamma}\right)$. The semistability of $x^{*}$ implies that $\left\|x_{k+1}-x^{*}\right\|=O\left(\left\|r_{k}\right\|\right)$ and this means that the quantity

$$
\frac{\left\|x_{k+1}-x^{*}\right\|}{\left\|x_{k+1}-x^{*}\right\|^{1+\gamma}+\left\|x_{k}-x^{*}\right\|^{1+\gamma}}=\frac{1}{\left\|x_{k+1}-x^{*}\right\|^{\gamma}+\frac{\left\|x_{k}-x^{*}\right\|^{1+\gamma}}{\left\|x_{k+1}-x^{*}\right\|}}
$$

forms a bounded sequence. Then this is possible if and only if there exists $\eta>0$ such that

$$
\frac{\left\|x_{k}-x^{*}\right\|^{1+\gamma}}{\left\|x_{k+1}-x^{*}\right\|} \geq \eta, \quad \forall k \geq 0
$$

or equivalently

$$
\left\|x_{k+1}-x^{*}\right\| \leq \frac{1}{\eta}\left\|x_{k}-x^{*}\right\|^{1+\gamma}, \quad \forall k \geq 0,
$$

which gives the superlinear convergence rate of $\left(x_{k}\right)$.

If $\gamma \geq 1$ then $\left\|r_{k}\right\|=O\left(\left\|x_{k+1}-x^{*}\right\|^{2}+\left\|x_{k}-x^{*}\right\|^{2}\right)$. The semistability of $x^{*}$ implies that

$$
\left\|x_{k+1}-x^{*}\right\|=O\left(\left\|r_{k}\right\|\right)
$$

this means that the quantity

$$
\frac{\left\|x_{k+1}-x^{*}\right\|}{\left\|x_{k+1}-x^{*}\right\|^{2}+\left\|x_{k}-x^{*}\right\|^{2}}=\frac{1}{\left\|x_{k+1}-x^{*}\right\|+\frac{\left\|x_{k}-x^{*}\right\|^{2}}{\left\|x_{k+1}-x^{*}\right\|}}
$$

forms a bounded sequence. Then this is possible if only if there exists $\delta>0$ such that

$$
\frac{\left\|x_{k}-x^{*}\right\|^{2}}{\left\|x_{k+1}-x^{*}\right\|} \geq \delta, \forall k \geq 0
$$

or equivalently

$$
\left\|x_{k+1}-x^{*}\right\| \leq \frac{1}{\delta}\left\|x_{k}-x^{*}\right\|^{2}, \forall k \geq 0
$$

which gives the quadratic convergence rate of $\left(x_{k}\right)$.

Let us note that in this second case where $\gamma \geq 1$ we find the same order of convergence than the inexact Newton method (see [26]). 
We now give a more general result for inexact Newton's subanalytic method where here the perturbation affects the multi-valued term. The problem we are interested in is the following:

$$
0 \in f\left(x_{k}\right)+\Delta f\left(x_{k}\right)\left(x-x_{k}\right)+\Omega\left(x_{k}, x-x_{k}\right)+F(x)
$$

where $\Omega: \mathbb{R}^{n} \times \mathbb{R}^{n} \rightarrow 2^{\mathbb{R}^{n}}$ is a given multifunction. This implies that the perturbation term appearing in (5) has to satisfy the inclusion $\omega_{k} \in$ $\Omega\left(x_{k}, x_{k+1}-x_{k}\right)$.

Theorem 4.2 Assume that $x^{*}$ be a semistable solution of (1) and let $\Omega: \mathbb{R}^{n} \times \mathbb{R}^{n} \rightarrow 2^{\mathbb{R}^{n}}$ be a multifunction satisfying the following assumptions:

- (INSM1) For each $x \in \mathbb{R}^{n}$ close to $x^{*}$, the variational inclusion

$$
0 \in f(x)+\Delta f(x) \eta+\Omega(x, \eta)+F(x+\eta)
$$

has a solution $\eta(x)$ such that $\eta(x) \rightarrow 0$ as $x \rightarrow x^{*}$.

- (INSM2) The estimate

$$
\|\omega\|=o\left(\|\eta\|+\left\|x-x^{*}\right\|\right)
$$

holds uniformly for $\omega \in \Omega(x, \eta), x \in \mathbb{R}^{n}$ and $\eta \in \mathbb{R}^{n}$ close enough to zero and satisfying

$$
0 \in f(x)+\Delta f(x) \eta+\omega+F(x+\eta)
$$

Then there exists $\delta \geq 0$ such that for any starting point $x_{0} \in \mathbb{R}^{n}$ close enough to $x^{*}$, there exists a trajectory $\left\{x_{k}\right\} \subset \mathbb{R}^{n}$ such that $x_{k+1}$ is a solution of (8) for each $k=0,1, \ldots$ satisfying

$$
\left\|x_{k+1}-x_{k}\right\| \leq \delta
$$

any such trajectory converges to $x^{*}$ and the rate of convergence is superlinear.

\section{Proof:}

Let us remark that the semistability of $x^{*}$ implies the existence of $\delta_{1}>0$ and $M>0$ such that for any $r \in \mathbb{R}^{n}$ and any solution $x(r)$ of the inclusion $r \in f(x)+F(x)$ satisfying $\left\|x(r)-x^{*}\right\| \leq \delta_{1}$ one has

$$
\left\|x(r)-x^{*}\right\| \leq M\|r\| .
$$


Now for any (fixed) $\left.\left.\delta_{2} \in\right] 0, \min \left(\frac{8}{11}, \delta_{1}\right)\right]$ and according to (INSM1), there exists $\delta \in\left(0, \frac{5}{8} \delta_{2}\right)$ such that the estimation $\left\|x_{k}-x^{*}\right\| \leq \frac{3}{5} \delta$ implies the existence of a solution $x_{k+1}$ of (8) satisfying $\left\|x_{k+1}-x^{*}\right\| \leq \delta_{2}$.

Let us remark that $x_{k+1}$ is a solution of the equation $r_{k} \in f(x)+F(x)$ with $r_{k}$ defined as in the proof of the previous theorem (see (8)) and with $\omega_{k} \in \Omega\left(x_{k}, x_{k+1}-x_{k}\right)$.

The estimations of $r_{k}$ given in the proof of the previous theorem and the one which is given for $\omega$ in (INSM2) imply that

$$
\left\|r_{k}\right\| \leq \frac{1}{4 M}\left(\left\|x_{k+1}-x_{k}\right\|+\left\|x_{k}-x^{*}\right\|\right)
$$

for sufficiently small value of $\delta_{2}$.

Since (9) holds with $r=r_{k}$ and $x\left(r_{k}\right)=x_{k+1}$ and taking in account the previous inequality, we obtain

$$
\begin{aligned}
\left\|x_{k+1}-x^{*}\right\| & \leq \frac{1}{4}\left(\left\|x_{k+1}-x_{k}\right\|+\left\|x_{k}-x^{*}\right\|\right) \\
& \leq \frac{1}{4}\left\|x_{k+1}-x^{*}\right\|+\frac{1}{2}\left\|x_{k}-x^{*}\right\|
\end{aligned}
$$

which yields

$$
\left\|x_{k+1}-x^{*}\right\| \leq \frac{2}{3}\left\|x_{k}-x^{*}\right\|
$$

which implies that

$$
\left\|x_{k+1}-x^{*}\right\| \leq \frac{2}{5} \delta
$$

and finally

$$
\left\|x_{k+1}-x_{k}\right\| \leq \delta
$$

It is easy to observe that if $\left\|x_{k}-x^{*}\right\| \leq \frac{3}{5} \delta$ and $\left\|x_{k+1}-x_{k}\right\| \leq \delta$, we obtain $\left\|x_{k+1}-x^{*}\right\| \leq \frac{8}{5} \delta \leq \delta_{2} \leq \delta_{1}$ and we can start again the procedure with $x_{k+1}$ and find the same estimations as in (10) and (11).

In this way, starting with a point $x_{0}$ satisfying $\left\|x_{0}-x^{*}\right\| \leq \frac{3}{5} \delta$, we find the next iterate $x_{1}$ solution of (8) for $k=0$ and satisfying $\left\|x_{1}-x^{*}\right\| \leq \frac{3}{5} \delta$. Thus, starting with $x_{1}$ we can find the next iterate $x_{2}$ solution of (8) for $k=1$ and satisfying $\left\|x_{2}-x^{*}\right\| \leq \frac{3}{5} \delta$. Repeating this argument, we show the existence of a trajectory $\left\{x_{k}\right\}$ such that for each $k, x_{k+1}$ is a solution of the variational inclusion (8) satisfying the inequality (11) which implies $\left\|x_{k+1}-x^{*}\right\| \leq \frac{3}{5} \delta$.

The convergence of the sequence comes from the inequality (10). 


\section{Acknowledgement}

We would like to thank the referees for valuable suggestions and comments that enabled us to improve the presentation of this paper.

\section{References}

[1] Aubin, J.-P.; Frankowska, H. (1990) Set Valued-Analysis. Birkhäuser, Boston.

[2] Aubin, J.-P. (1984) "Lipschitz behaviour of solutions to convex minimization problems", Math. Oper. Res. 9(1): 87-111.

[3] Bierstone, E.; Milman, P.D. (1988) "Semianalytic and subanalytic sets", IHES Publications Mathématiques 67(1): 5-42.

[4] Bochnak, J.; Coste, M.; Roy, M.-F. (1988)Géométrie Algébrique Réelle. Springer, Berlin.

[5] Bolte, J.; Daniilidis, A.; Lewis, A.S. (2009) "Tame mapping are semismooth", Math. Program. 117(1-2): 5-19.

[6] Bonnans, J.-F. (1994) "Local Analysis of Newton-Type Methods for Variational Inequalities and Nonlinear Programming", Appl. Math. Optim. 29(2): 161-186.

[7] Burnet, S.; Jean-Alexis, C.; Piétrus, A. (2012) "A multipoint iterative method for semistable solutions", Appl. Math. E-notes 12: 44-52.

[8] Burnet, S.; Jean-Alexis, C.; Piétrus, A. (2011) "An iterative method for semistable solutions", RACSAM 105(1): 133-138.

[9] Burnet, S.; Piétrus, A. (2011) "Local analysis of a cubically convergent method for variational inclusions", Appl. Mat. 38(2): 183-191.

[10] Cabuzel, C.; Piétrus, A. (2008) "Local convergence of Newton's method for subanalytic variational inclusions", Positivity 12(3): 523533.

[11] Cabuzel, C.; Piétrus, A. (2009) "Solving variational inclusions by a method obtained using a multipoint formula", Rev. Mat. Complut. 22(1): $63-74$. 
[12] Clarke, F.H. (1990) Optimization and Nonsmooth Analysis. Society for Industrial and Applied Mathematics, Philadelphia PA.

[13] Dedieu, J.-P. (1992) "Penalty functions in subanalytic optimization", Optimization 26(1-2): 27-32.

[14] Dontchev, A.L.; Hager, W.W. (1994) "An inverse function theorem for set-valued maps", Proc. Amer. Math. Soc. 121: 481-489.

[15] Dontchev, A.L. (1996) "Local convergence of the Newton method for generalized equation", C.R.A.S. 322(1): 327-331.

[16] Dontchev, A.L. (1996) "Uniform convergence of the Newton method for Aubin continuous maps", Serdica Math. J. 22(1): 385-398.

[17] Dontchev, A.L. (1996) "Local Analysis of a Newton-type method based on partial linearization", in: J. Renegar et al. (Eds.) The Mathematics of Numerical Analysis. 1995 AMS-SIAM Summer Seminar in Applied Mathematics, Providence RI: AMS., Lect. Appl. Math. 32: 295-306.

[18] Dontchev, A.L.; Quincampoix, M.; Zlateva, N. (2006) "Aubin criterion for metric regularity", J. of Convex Analysis 13(2): 281-297.

[19] Dontchev, A.L.; Rockafellar, R.T. (2009) Implicit Functions and Solution Mappings. Springer Monographs in Mathematics.

[20] Ferris, M.C.; Pang, J.S. (1997) "Engineering and economic applications of complementary problems", SIAM Rev. 39(4): 669-713.

[21] Gabrielov, A.M. (1968) "Projection of semianalytic sets", Funkcional. Anal. Prilozen. 2(4): 18-30.

[22] Geoffroy, M.H.; Hilout, S.; Pietrus, A. (2003) "Acceleration of convergence in Dontchev's iterative method for solving variational inclusions", Serdica Math. J. 29(1): 45-54.

[23] Geoffroy, M.H.; Pietrus, A. (2003) "A superquadratic method for solving generalized equations in the Hölder case", Ricerche Mat. 52(2): 231-240.

[24] Geoffroy, M.H.; Hilout, S.; Pietrus, A. (2006) "Stability of a cubically convergent method for generalized equations", Set-Valued Anal. 14(1): $41-54$. 
[25] Hironaka, N. (1973) "Subanalytic sets", Number Theory, Algebraic Geometry and Commutative Algebra, in honour of Y. Akizuki Kinokuniya, Tokyo: 453-493.

[26] Izmailov, A.F.; Solodov, M.V. (2010) "Inexact Josephy-Newton framework for generalized equations and its applications to local analysis of Newtonian methods for constrained optimization", Comput. Optim. Appl. 46: 347-368.

[27] Jean-Alexis, C. (2006) "A cubic method without second order derivative for solving variational inclusions", C. R. Acad. Bulgare Sci. 59(12): 1213-1218.

[28] Lojasiewicz, S. (1964) Ensembles Semi-Analytiques. IHES, Mimeographed notes.

[29] Mordukhovich, B.S. (1993) "Complete characterization of openness metric regularity and lipschitzian properties of multifunctions", Trans. Amer. Math. Soc. 340: 1-36.

[30] Mordukhovich, B.S. (2006) Variational Analysis and Generalized Differentiation. I: Basic Theory. Vol. 330, Springer.

[31] Piétrus, A. (2000) "Generalized equations under mild differentiability conditions", Rev. Real. Acad. Ciencias de Madrid 94(1): 15-18.

[32] Piétrus, A. (2000) "Does Newton's method for set-valued maps converges uniformly in mild differentiability context?", Rev. Colombiana. Mat. 32: 49-56.

[33] Qi, L.; Sun, J. (1993) "A nonsmooth version of Newton's method", Math. Program. 58: 353-367.

[34] Robinson, S.M. (1979) "Generalized equations and their solutions, part I: basic theory", Math. Program. Study 10: 128-141.

[35] Robinson, S.M. (1980) "Strongly regular generalized equations", Math Oper. Res. 5: 43-62.

[36] Robinson, S.M. (1982) "Generalized equations and their solutions, part II: Application to nonlinear programming", Math. Program. Study 19: 200-221. 
[37] Rockafellar, R.T. (1984) "Lipschitzian properties of multifonctions", Nonlinear Analysis 9: 867-885.

[38] Rockafellar, R.T.; Wets, R.J.B. (1988) Variational analysis. A Series of Comprehensives Studies in Mathematics 317, Springer.

[39] Van Den Dries, L.; Miller, C. (1996) "Geometric categories and ominimal structures", Duke Math. J. 84: 497-540. 
